\title{
L'univers esthétique des photographies de Cy Twombly et le renouvellement du regard sur
} son œuvre

The aesthetic of Cy Twombly's photographs and new ways of looking at his work

\section{Chiara Agradi}

\section{CpenEdition}

\section{Journals}

Édition électronique

URL : http://journals.openedition.org/cel/581

DOI : $10.4000 /$ cel.581

ISSN : 2262-208X

Éditeur

École du Louvre

Référence électronique

Chiara Agradi, «L'univers esthétique des photographies de Cy Twombly et le renouvellement du regard sur son œuvre », Les Cahiers de l'École du Louvre [En ligne], 10 | 2017, mis en ligne le 03 mai 2017, consulté le 17 septembre 2019. URL : http://journals.openedition.org/cel/581 ; DOI : 10.4000/cel.581

Ce document a été généré automatiquement le 17 septembre 2019.

\section{(c) (†) $\ominus$}

Les Cahiers de l'École du Louvre sont mis à disposition selon les termes de la licence Creative Commons Attribution - Pas d'Utilisation Commerciale - Pas de Modification 4.0 International. 


\title{
L'univers esthétique des photographies de Cy Twombly et le renouvellement du regard sur son œuvre
}

The aesthetic of Cy Twombly's photographs and new ways of looking at his work

\author{
Chiara Agradi
}

Cy Twombly est l'un des artistes américains les plus influents du vingtième siècle. Malgré sa réputation de peintre et sculpteur, il a constitué tout au long de sa vie un vaste corpus photographique, qui est cependant demeuré inconnu jusqu'aux années 1990. En effet, si d'une part ce médium intéresse l'artiste depuis ses débuts, d'autre part sa relation avec l'appareil photographique s'inscrit dans une dimension intime de recherche personnelle. N'ayant pas voulu rendre publique son œuvre photographique pendant longtemps, un travail de valorisation reste encore à accomplir. La grande majorité de ses photographies ont été prises entre les années 1970 et 2011, surtout en Italie, à Rome et à Gaeta, village pittoresque où il déménage à la fin des années 1950. Il s'agit de Polaroïds qui étaient, dans un deuxième temps, imprimés par le biais d'une photocopieuse couleur; la technique d'impression avait été développée en collaboration avec la maison d'édition Schirmer/Mosel, qui a aussi participé activement à la valorisation de ce corpus grâce à la publication de quatre catalogues raisonnés de photographie. À la mort de Twombly, en 2011, son ami et collaborateur Nicola del Roscio a investi ses efforts dans l'institution de la Fondation éponyme, qui vise désormais à la conservation et à la valorisation de ce corpus photographique. Le fonds est regroupé dans cette Fondation et consiste en un vaste ensemble soigneusement conservé, qui n'avait jusqu'à présent pas fait l'objet d'une étude scientifique. En effet, malgré la publication des quatre catalogues raisonnés, un grand nombre de photographies demeurent inédites dans les archives de la Fondation.

2 La récente redécouverte du fonds et le travail académique qui a suivi ont permis une transformation du regard porté sur l'œuvre de l'artiste. Aujourd'hui, nous assistons à 
une valorisation significative de la figure de Cy Twombly photographe, et nombre d'expositions internationales sont entièrement consacrées à son travail photographique. Ceci représente le dernier défi de Cy Twombly, qui s'est cependant intéressé à la photographie pendant toute sa carrière. Néanmoins, la notoriété universelle de son œuvre picturale tend à désigner l'artiste comme peintre, en négligeant le rôle que la photographie a joué dans sa carrière. Loin d'être un simple outil technique, la photographie constitue pour Cy Twombly un médium d'investigation scientifique et esthétique qui lui permet d'opérer une relecture de son œuvre picturale et sculptée et du monde qui l'entoure. Ainsi, les virtuosités de la technique lui permettent de réfléchir à la fois à la transformation esthétique que la vision photographique opère sur les objets et ses spécificités, et à la transformation physique de l'objet photographique lui-même, que Twombly présente sous forme de Polaroïd et d'impression sur papier mat dry-print. C'est donc en raison de la réticence de Twombly que la première exposition de ses photographies n'eut lieu qu'en 1993 à la Matthew Marks Gallery à New York, environ quarante ans après la date d'exécution des premiers clichés. Les photographies exposées à la Matthew Marks Gallery en 1993 sont des impressions couleur exécutées artisanalement par l'Atelier Fresson à partir de photographies instantanées. L'auteur, qui ne fut jamais enthousiasmé par le résultat obtenu par l'atelier français, s'adressa dans un deuxième temps à Lothar Schirmer, éditeur munichois qui se chargea, dès les années 2000, de l'impression dry-print des Polaroïds. La collaboration entre Cy Twombly et la maison d'édition Schirmer/Mosel a d'ailleurs été déterminante pour la valorisation et la fortune commerciale des photographies, dont l'impression avait été congelée pendant plus d'une décennie.

\section{Du Black Mountain College à Rome : les premières rencontres avec la photographie}

3 La rencontre féconde de Cy Twombly avec la photographie remonte au début des années 1950. En 1952, Cy Twombly passe l'été au Black Mountain College avec son ami Robert Rauschenberg. Dans ce cadre, les deux artistes exécutent des photographies, des peintures et des assemblages sculptés, à la palette généralement très restreinte. Un tel choix n'est pas qu'une coïncidence. En effet, il se trouve qu'à l'époque du séjour de Twombly au Black Mountain College, une réflexion critique et artistique autour de l'usage et de la valeur de la peinture noir et blanc était menée par les enseignants et les critiques de l'époque, d'Aaron Siskind à Clement Greenberg. C'est à ce moment que remonte un des premiers clichés significatifs de Cy Twombly, Still Life, Black Mountain College, NC, 1951 (fig. 1). Il s'agit d'une nature morte au sens classique du terme, qui rend aussi hommage, à l'instar des impressions de Michael Krüger', à l'œuvre de Giorgio Morandi, par la simplicité de la composition et la pureté des formes. La photographie est animée par la réfraction de la lumière sur le verre, qui semble taché par de la gouache ou de l'acrylique. Un lien subtil est tissé entre couleur et absence de couleur: s'agissant d'une photographie datée de 1951, il est vraisemblable que la gouache dont ces bouteilles sont tachées soit noire ou blanche. Ce cliché serait donc le témoin de la tendance esthétique du Black Mountain College et de la sensibilité de l'artiste aux interactions entre photographie et peinture, notamment peinture en noir et blanc. 
Fig. 1

La Fondation Nicola del Roscio n'ayant pas donné l'autorisation de publier cette image, elle est visible sur cette page:

http://lunettesrouges.blog.lemonde.fr/files/2011/07/a-Twombly-Still-Life-Black-Mountain-

College-1951.jpg

Cy Twombly, Still Life, 1951, Black Mountain College, NC, impression dry-print sur carton,

$43,1 \times 27,9 \mathrm{~cm}$

Si l'expérience au Black Mountain constitue un moment important pour la sensibilisation de Cy Twombly, le Grand Tour que l'artiste entreprend avec Robert Rauschenberg en Europe la même année marque un véritable tournant dans la conception de son œuvre photographique. Le voyage est assidûment documenté par un corpus de photographies prises par Rauschenberg, qui témoignent d'un intérêt conjoint des artistes pour certaines techniques photographiques. En guise d'exemple, une série de cinq clichés, qui porte le nom de Cy and Roman Steps, semble attester de la curiosité des deux artistes pour la chronophotographie à la Muybridge. Il s'agit d'une série de clichés qui montrent Twombly en train de descendre les escaliers en face de l'église de Santa Maria in Aracoeli. Ces photographies, habitées par le dynamisme et le goût de l'expérimentation en elle-même, montrent à quel point Rauschenberg, et par conséquent Twombly, s'intéressait peu aux vues pittoresques de la ville de Rome. Ainsi, un certain goût pour le fragment et pour l'objet trouvé ressort des photographies de Rauschenberg. En effet, si Twombly manifestait son intérêt pour le fragment et les ruines antiques à travers des cahiers de dessins animés par une attitude réflexive et dédiée à l'étude et à la connaissance de Rome et de son patrimoine, Rauschenberg semble avoir davantage exploré cette thématique muni de son appareil photographique. $C y+B o b$, Venice, 1952 (fig. 2) est un autre cliché iconique de leur Grand Tour, réalisé au moment où ils remontent à Venise après deux semaines passées à Rome. Encore une fois, nous ne sommes pas confrontés à une photographie documentaire, mais à un élégant montage où les aspects de simultanéité et de dynamisme sont explorés grâce à un jeu de superposition. Le thème du fragment, aussi bien que celui de l'antique, sont explorés dans la représentation fragmentée et multiple des chevaux de la basilique de San Marco, dont la surface en bronze renvoie à la statuaire classique grecque. Cy Twombly y apparaît à droite, le regard absorbé et concentré comme celui d'un savant. De nouveau, si Rauschenberg est dans l'action, prend des photographies et documente assidûment le voyage, Twombly représente son contrepoids. Il vit le voyage en Europe comme une expérience formative qui nécessite à la fois dévouement et étude. Il ne s'agit pas ici d'affirmer l'existence d'un lien direct entre le travail de Rauschenberg et celui de Twombly; toutefois il est possible d'avancer des hypothèses sur les origines de l'activité photographique de Twombly, en recherchant des indices dans le contexte culturel dans lequel il s'insère dès qu'il quitte la Virginie, son pays d'origine, pour New York, à la découverte des dernières tendances esthétiques. Quant à l'influence de Rauschenberg, qui était à l'époque familier de l'outil photographique, il est vraisemblable que le fait d'avoir entrepris avec lui un long voyage en Europe ait pu avoir des conséquences sur le rapport de Twombly à la photographie. 
Fig. 2

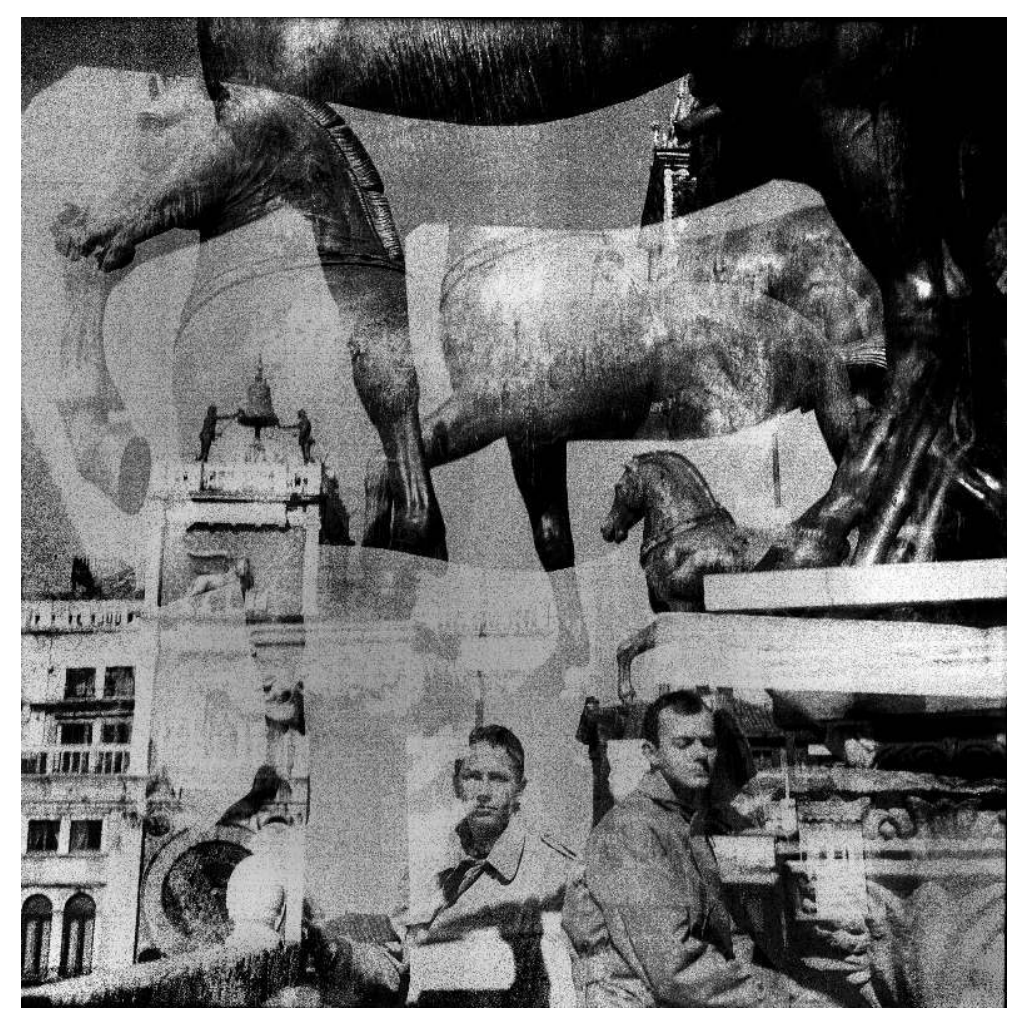

Robert Rauschenberg, Bob + Cy, Venice, 1952, tirage gélatino-argentique, $38.1 \times 38.1 \mathrm{~cm}$ (15 × 15 inches)

(c) Robert Rauschenberg Foundation

5 Le voyage en Europe, notamment à Rome, investit l'œuvre de Twombly de ce que Roland Barthes définit par « l'effet méditerranéen ${ }^{2}$ ». Tel est l'esprit qui anime l'œuvre de Twombly avant même son déplacement définitif en Italie à la fin des années 1970 car, comme l'a dit Barthes à ce propos, reprenant un discours de Paul Valéry, " Il arrive que l'avenir soit cause du passé ${ }^{3}$ ». En effet, puisqu'il avait grandi dans le climat doux et l'architecture classique de sa région d'origine, la Virginie, dans le sud des États-Unis, l'Italie lui semblait la destination la plus naturelle. Par « effet méditerranéen ", Barthes désigne donc un ensemble de souvenirs et de sensations, une sensibilité à la culture et à la langue grecque et latine, une curiosité pour la mythologie et la poésie et une sensibilité aux couleurs de la nature. La lumière intense, celle du soleil qui chauffe les façades jaunâtres des maisons du centre de Rome, celle crépusculaire qui s'éteint sur la mer de la baie de Gaeta, où il vit pendant sa vieillesse, ou encore celle poussiéreuse de ses appartements aux murs immaculés, a également un fort impact sur la production des années italiennes de Twombly, qui a recours à l'appareil photographique de plus en plus systématiquement.

\section{Photographies en transformation : du Polaroïd au dry- print}

6 La diffusion et l'exposition des photographies de Twombly commencent très tard, et cela découle également de l'incessante recherche de l'artiste les supports et sur les techniques d'impression. En effet, ce n'est qu'après plusieurs années d'essais que 
Twombly met au point sa technique distinctive: il imprime ses photographies sur papier, non pas par un processus artisanal de photogravure, mais avec la technique dryprint, qui consiste en une version spécialisée de la simple photocopie couleur. La photocopieuse originelle employée par Cy Twombly se trouve aujourd'hui dans les espaces de la maison d'édition Schirmer/Mosel. Achetée au début des années 2000, elle garde le secret du processus d'impression des Polaroïds, de leur aspect mat et chaud, obtenu après des années d'expérimentation. En effet, les Polaroïds occupent pour Twombly la place de la partition musicale pour le musicien: leur impression, le moment où les couleurs froides des Polaroïds éclatent sur la surface du papier, correspond à l'acte de la performance des notes imprimées sur la partition musicale.

7 Cy Twombly manifeste son désir d'imprimer les Polaroïds sur papier au début des années 1990. En effet, c'est en 1993 que la galerie Matthew Marks, située au 1018 Madison Avenue, New York, organise la première exposition consacrée à vingt-neuf tirages exécutés par l'Atelier Fresson, à Savigny-sur-Orge. Le photographe commence à collaborer avec Michel et Jean-François Fresson en 1990. La particularité des tirages exécutés avec le procédé Fresson réside dans les effets de pointillisme de la surface imprimée et dans la nature très stable des pigments naturels. À l'époque de l'exposition à la Matthew Marks Gallery, les tirages Fresson étaient ceux dont les couleurs étaient les plus stables et permanentes. Cependant, Cy Twombly n'a jamais été enthousiasmé par le résultat obtenu. Insatisfait par le procédé Fresson et probablement effrayé par le jugement mordant du public de New York, Cy Twombly semble ne pas vouloir exposer ni valoriser ses photographies. Ce n'est qu'au tournant des années 2000 que l'artiste trouve dans un copyshop de Lexington une photocopieuse dont les impressions satisfont ses attentes. Il obtient des tirages à son goût pour le prix modeste de 25 centimes de dollar. Néanmoins, son enthousiasme s'éteint bientôt, car le commerce périclite à la mort prématurée des propriétaires et la photocopieuse disparaît. Démotivé par la perte, Cy Twombly ne semble pas pour autant partir à la recherche d'une solution de remplacement. Après tout, les tirages à 25 centimes de dollar n'étaient que de simples copies couleur. En revanche, la qualité et le grain du papier du copyshop de Lexington semblaient irremplaçables.

8 C'est grâce à l'initiative de Lothar Schirmer, qui se lance à la recherche d'une photocopieuse de substitution, que le processus d'impression se systématise au début des années 2000. Quant à la photocopieuse, il s'agit d'une machine aux poudres colorées qui nécessite des soins particuliers. Cy Twombly trouve en Schirmer/Mosel non seulement son atelier d'impression photographique, mais aussi la maison d'édition qui publiera les quatre catalogues raisonnés qui ont vu le jour jusqu'à présent. Lothar Schirmer et son équipe ont travaillé en symbiose avec Cy Twombly autour de la production photographique de 2002 à 2011. Ils ont fixé le nombre d'éditions à un maximum de six et administré les ventes. En ce qui concerne l'organisation du travail, l'artiste se rendait à Munich avec des boîtes de Polaroïds, où il procédait, avec Lothar Schirmer, à une sélection des meilleurs tirages et à l'impression de ces derniers. En même temps, Schirmer organisait les catalogues raisonnés sur la base des photographies choisies en accord avec l'artiste. Ainsi, une fois l'impression terminée, la dernière étape du processus était la signature des impressions.

9 L'impression des Polaroïds, sur un papier de format standard $43.1 \times 27,9 \mathrm{~cm}$ dont l'épaisseur gênait souvent le fonctionnement de la photocopieuse, était un processus d'expérimentation empirique. L'impression était effectuée par essais successifs, en 
réglant au fur et à mesure les tons chromatiques. Les impressions de Cy Twombly se caractérisent par des bavures de couleur, des effets de tachisme, des marques d'impression et de sédimentation de poudres de couleur. Certaines de ces impressions ont été conservées comme épreuves d'artiste ; d'autres, non moins imparfaites, ont été élevées par Twombly au rang d'œuvres. La notion d'erreur dans le processus d'impression est donc aléatoire. Du point de vue du résultat, l'aspect des tirages issus de la photocopieuse de Schirmer/Mosel s'apparente au résultat coloriste artisanal des tirages au procédé Fresson. Le pigment en poudre constitue l'un des éléments fondamentaux du rendu esthétique des impressions de Cy Twombly. Leurs tons chauds, obtenus grâce au mélange des pigments en poudre avec la silicone, étaient surprenants par rapport à la surface brillante et aux tons froids des Polaroïds. Une vraie transformation s'opère dans la photographie de Cy Twombly, pour qui le Polaroïd ne représente qu'une étape intermédiaire au sein d'un processus plus complexe d'achèvement de l'image photographique.

\section{Vision photographique et vision optique : les enjeux esthétiques de la photographie de Cy Twombly}

10 La maturité de Cy Twombly est donc caractérisée par l'omniprésence du Polaroïd SX-70, par l'assiduité de la production photographique et, à partir des années 2000, par l'impression et l'élaboration des catalogues raisonnés. Inspiré par le climat tempéré et la lumière chaude de l'Italie, Twombly semble avoir une préférence pour les paysages méditerranéens, notamment le village pittoresque de Gaeta - où il installe son atelier - et le golfe de Naples, ainsi que pour les objets qui habitent les chambres de ses appartements romains. Dans tous les cas, Twombly manifeste tout au long de sa carrière un refus assez évident de la belle forme. Cela se traduit par la fragmentation systématique du sujet photographié et par une approche méditative qui néglige les règles de prise de vue et de composition classiques. Le fragment, dispositif de vision auquel Twombly se sensibilise dès lors qu'il découvre les restes du Colosse de Constantin conservés dans la cour du musée du Capitole à Rome, le découpage de l'objet, et la subordination de son intégrité à la force expressive de l'œuvre constituent les éléments clés de sa recherche sur l'image. Ainsi, la prédilection pour des compositions fragmentaires se justifie par les caractéristiques propres à l'appareil photographique qui impose, par opposition à l'œil qui bouge, un cadre défini impossible à dépasser et qui est, en soi, un fragment de réalité. La photographie rend compte d'une réalité autre que celle de l'œil en donnant vie à un nouveau vocabulaire de signes et de formes, à une nouvelle écriture. De même, quand Twombly représente un objet, il lui confère un sens nouveau, et cela d'autant plus que l'aspect fragmentaire en est insaisissable par l'œil (car seul l'appareil photographique nous offre une vision fragmentée d'un objet entier).

11 La photographie participe donc à la perte de correspondance entre l'objet et sa représentation; elle s'insère dans la relation univoque qui existe, selon les mots de Derrida $^{5}$, entre signifiant et signifié, en créant un nouveau langage. C'est dans les clichés de nature morte que la transformation des objets et leur emploi au service d'une recherche esthétique plus profonde que la simple quête d'une belle composition s'exprime dans sa forme la plus accomplie. En effet, il se trouve qu'à partir des années 1990 une partie de la recherche de Twombly à Gaeta est systématiquement consacrée à 
l'étude d'objets inanimés, principalement organiques, disposés de façon arbitraire sur une table. Nous devons imaginer Cy Twombly aux prises avec des objets ordinaires, comme de la nourriture trouvée sur la table à manger ou encore des fleurs coupées et fanées abandonnées sur une surface plane et poussiéreuse. Simples en apparence, ces clichés cachent une complexité inattendue.

Par ailleurs, les évocations du genre de la nature morte dans la tradition picturale et photographique, et la complexité technique de ces clichés rendent ces épreuves parmi les plus intéressantes de la production de Twombly. Si nous prenons comme exemple Lemons, Gaeta, 2005 (fig. 3), nous sommes immédiatement frappés par deux éléments : les citrons sont à peine reconnaissables à cause du flou et le cadre très rapproché ne nous donne qu'une vision fragmentaire des objets de la composition. Le fragment s'inscrit dans l'œuvre de Twombly, il lui appartient. Les raisons pour lesquelles Twombly adopte le fragment comme dispositif de vision sont diverses; héritage de la culture visuelle de notre époque, la vision fragmentaire découle aussi d'une recherche spécifique de Twombly sur l'objet. Dans Lemons, Gaeta, 2005, le caractère incomplet de la composition nous plonge dans le temps immanent à ces clichés qui sont la représentation d'un instant, d'un fragment de temps. Quoique le genre de la nature morte nous suggère traditionnellement un calme éternel qui sous-entend un temps statique et immuable, la fragmentation de l'image de la part de Twombly comporte une réévaluation de la temporalité de l'œuvre: la fragmentation affecte et transforme la perception du temps.

Fig. 3

La Fondation Nicola del Roscio n'ayant pas donné l'autorisation de publier cette image, elle est visible sur cette page :

http://lunettesrouges.blog.lemonde.fr/files/2011/07/a-Twombly-Lemosn-Gaeta-2005.jpg

Cy Twombly, Lemons, Gaeta, 2005, impression dry-print sur carton, 43,1 x 27,9 cm

Twombly exploite volontairement les erreurs que le photographe peut - malgré lui commettre, et bouleverse les règles communes de la photographie. Surimpressions, anamorphoses et décadrage font partie de son vocabulaire expressif. Les objets immortalisés par Cy Twombly subissent une véritable transfiguration car, privés de leur matérialité, ils survivent uniquement par leurs formes floues et leurs couleurs, formant avec les autres éléments du cadre une composition qui tend à l'abstraction. La lumière joue aussi un rôle clé dans le processus de transformation du sujet. Comme le dit Hubertus von Amelunxen, dans la préface au catalogue raisonné de photographies de Cy Twombly:

L'œuvre incomparable de Cy Twombly se fonde sur un art de la lumière. Son art est porteur de lumière. Dans ses peintures et ses dessins la lumière fait disparaitre toute autre signification dans le fond, dans la couleur et dans le mouvement qui ouvre, elle foule et épaissit des doutes pour que sorte une splendeur définitive simple.

La transformation de l'objet photographique et les exploitations des erreurs techniques sont au cœur de la démarche de Cy Twombly. Les natures mortes nous en donnent un exemple, mais c'est dans les séries de photographies consacrées à la peinture et à la sculpture que ce processus de transformation de l'objet s'accomplit. La photographie permet à Twombly de prendre du recul, de fuir la relation physique qui s'instaure entre l'artiste et la toile pour investiguer la surface peinte par la lentille de l'objectif, à une certaine distance. Ainsi, l'appareil photo qui se dresse entre l'œil de l'artiste et l'œuvre 
achevée peut être interprété comme une couche qui se superpose à la surface peinte, car c'est en regardant le cliché que nous parvenons à la lecture que Twombly veut nous donner de son œuvre.

\section{Entre photographie et peinture, le plafond du Louvre, testament esthétique de l'artiste}

Collectionneur d'art et de sculpture ancienne, Twombly tourne souvent l'objectif vers les œuvres d'art qu'il possède, et qui sont exposées dans les chambres de ses habitations ; il exécute aussi des clichés de ses propres toiles et sculptures. Les séries consacrées à la peinture, notamment Painting detail, Gaeta, 2000 (fig. 4), rendent visibles des caractéristiques matérielles de l'œuvre, les craquelures de la couleur et la trame de la toile. Le flash est tourné vers la surface peinte de la toile, résultat d'un geste aveugle, où la main précède l'œil. Le hasard lumineux participe aussi à donner un aspect fortuit au cliché, qui immortalise le fruit hasardeux d'un rayon de lumière rasante, ou directe, qui frappe la surface peinte et met en valeur des détails qui échappent à l'œil et qui amènent à une relecture de l'œuvre. Ici encore, l'élément qui s'interpose entre l'appareil photographique et la surface peinte des œuvres et qui en change radicalement la perception, c'est - comme l'a dit Hubertus von Amelunxen - la lumière. Il est pertinent de retrouver dans cette photographie de Cy Twombly la notion d'inconscient optique telle qu'elle est proposée par Benjamin ${ }^{7}$ : l'appareil photo de Twombly rend visible ce qui échappe à la vision optique, et nous confronte à une lecture presque scientifique de l'œuvre, telle que nous pourrions l'obtenir dans un laboratoire d'analyse ou de restauration d'œuvres d'art. Quand la lumière aveuglante du flash épouse la désactivation intentionnelle de la fonction autofocus de l'appareil, le résultat est une composition qui se rapproche de l'abstraction; les détails deviennent méconnaissables et le dessin succombe aux plus audacieux effets de colorisme.

Fig. 4

La Fondation Nicola del Roscio n'ayant pas donné l'autorisation de publier cette image, elle est visible sur cette page

https://artblart.files.wordpress.com/2012/04/cy-twombly-painting-detail-and-by-the-ionian-seasculpture-bassano-in-teverina-dryprint-on-cardboard-1992-web.jpg

Cy Twombly, Painting Detail and "By the Ionian Sea" Sculpture, Bassano in Teverina, 1992, impression dry-print sur carton, $43,1 \times 27,9 \mathrm{~cm}$

La force de la photographie par rapport à la peinture consiste à pouvoir remettre en question les notions d'orientation et de cadrage, et c'est aussi sur ces thèmes que l'artiste réfléchit. Lorsque Twombly réalise une œuvre picturale, il lui donne une orientation précise, celle de l'accrochage, mais nous gardons une marge de mouvement qui nous permet, par exemple, de nous placer à côté de la toile et d'observer l'épaisseur des couches de couleur. L'aplatissement auquel la photographie soumet les objets immortalisés transforme les toiles en surfaces pures. La photographie en tant qu'objet n'est qu'une surface où formes et couleurs plus ou moins abstraites se déploient, en dépit des valeurs de ressemblance et de composition. L'œuvre photographique de Cy Twombly est donc un champ de possibilités interprétatives, le genre d'œuvre qu'Umberto Eco définit comme opera aperta ${ }^{8}$, un champ d'action et de possibilité, d'éléments en transformation perpétuelle. 
17 La commande officielle du musée du Louvre, en 2010, est un moment clé dans la carrière de l'artiste. Il conçoit alors la décoration du plafond de la salle des bronzes grecs, située dans la partie la plus ancienne du musée, l'aile Lescot. Une évidence s'impose devant ce plafond : il ne ressemble à peu près à rien de ce que Twombly avait peint jusque-là, car il a dû s'adapter au lieu, et non s'imposer. En revanche, comme c'est souvent le cas, une relation subtile mais profonde existe entre l'œuvre picturale de l'artiste et l'esthétique de ses photographies. Pour répondre à la commande, Twombly fait d'abord face aux contraintes du plafond, caractérisé par sa forme très allongée. Afin de contrebalancer l'immensité de la surface plane, il conçoit des cercles de différentes couleurs, qui sont pour lui des boucliers mais qui peuvent aussi être perçus comme des planètes qui tournent dans l'immensité du ciel bleu. Ces formes sphériques flottent dans l'espace léger du ciel bleu Giotto et donnent au plafond un effet de mouvement. Aux sphères se superposent sept noms de sculpteurs grecs dans des cartouches blancs : Phidias, Myron, Lysippe, Praxitèle, Polyclète, Céphisodote, Scopas. Un degré certain de définition et de précision des formes caractérise le style du plafond et s'éloigne de la plupart des œuvres picturales de l'artiste. En revanche, l'évocation de l'univers classique de la Grèce antique trouve sa justification dans l'influence de la culture méditerranéenne sur l'œuvre de Twombly. Le bleu du plafond, qui évoque à la fois la couleur du ciel et de la mer, renvoie aux couleurs vives du golfe du Naples, qu'il apercevait de la fenêtre de son atelier à Gaeta. En effet, les séries de photographies qui portent le titre de Miramare ont pour sujet la plage de Gaeta et son golfe, et se caractérisent par leurs couleurs saturées et le rendu tactile de l'atmosphère : la mer et le ciel se fondent dans une surface unique et lumineuse. Le plafond monochrome du Louvre, même s'il est différent du point de vue stylistique, s'apparente à cette recherche esthétique, qui témoigne des interactions constantes entre photographie et peinture dans l'œuvre de Cy Twombly. Ainsi, certains boucliers peints sont représentés coupés, le cadre de la composition étant ouvert et libre. Ce qui nous suggère que lorsqu'il est confronté à une surface plane et bidimensionnelle, Twombly a recours à des dispositifs esthétiques propres à la vision photographique. En effet, son désintéressement pour la composition équilibrée nous permet de réfléchir tant à l'opposition entre vision optique et vision photographique, qu'au positionnement de l'artiste face à cet enjeu esthétique. Selon Rosalind Krauss ${ }^{9}$, dont les théories se fondent sur celles de son prédécesseur Walter Benjamin, la photographie dévoile des aspects de la vision qui ne sont pas propres à la vision optique. Ce que Krauss appelle donc inconscient optique sont les modalités de vision étrangères à l'œil (agrandissement, réduction, fragmentation, détail), rendues possibles par l'appareil photographique, qui en est l'extension physique. La photographie «dévoile [...] les aspects physiognomoniques, les mondes d'images qui habitent les plus petites choses suffisamment expressifs, suffisamment secrets pour avoir trouvé abri dans les rêves éveillés, mais qui, ayant changé d'échelle, devenus énonçables, font désormais clairement apparaître la différence entre technique et magie comme une variation historique $^{10}$ ». Il est évident qu'une surface si grande impose une recherche et une organisation de l'espace préalables; néanmoins, plus significative encore est l'accentuation de la bidimensionnalité structurelle du plafond, et l'importance accordée par Twombly à cet aspect. En effet, il conçoit la surface du plafond comme s'il s'agissait de la surface plane et précadrée du papier photographique. Le traitement de l'espace s'éloigne du modèle traditionnel de la peinture de plafond: il n'y a pas de figure au centre, ni de division de l'espace en cadres symétriques, l'espace est traité 
comme un champ ouvert, où les limites structurelles ne constituent cependant pas le cadre de la composition.

Fig. 5

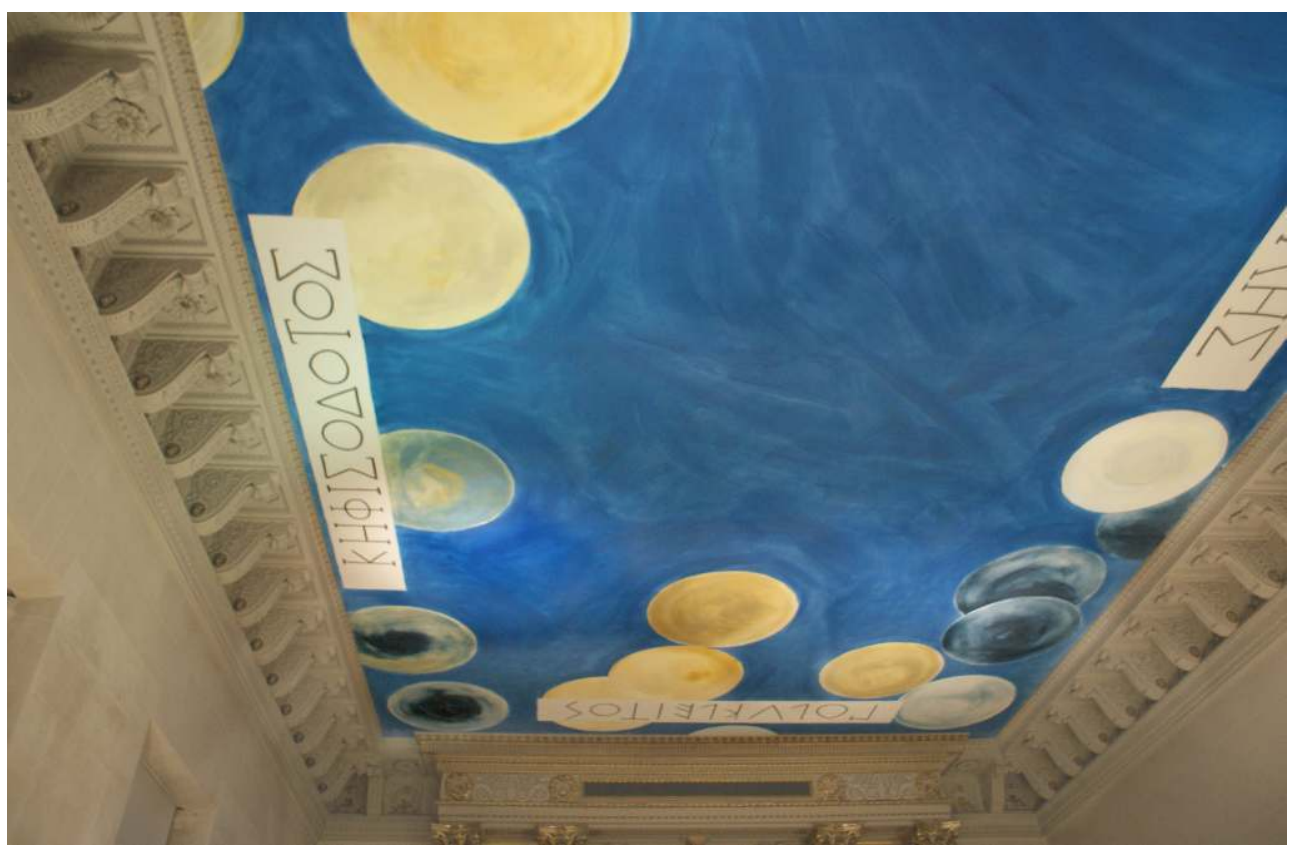

CY TWOMBLY, VUE DU PLAFOND DE LA SALLE DES BRONZES DU MUSÉE DU LOUVRE

(C) PHOTOgRAPHIE : ML / ÉCOLE DU LOUVRE

Ce n'est pas une coïncidence si Cy Twombly s'engage dans la décoration d'une salle destinée à conserver et montrer des sculptures et des objets manufacturés. Il manifeste tout au long de sa carrière un intérêt pour la sculpture et ses propriétés. Si ses assemblages sculptés couverts de plâtre et de gouache blanche sont très connus, c'est moins le cas des séries de photographies qui ont pour sujet des sculptures anciennes de sa collection privée, et ses propres sculptures. Twombly s'intéresse à la sculpture pour ses qualités intrinsèques. Une sculpture est essentiellement ambiguë car elle n'est pas immédiatement lisible dans sa totalité ; elle est compréhensible de différentes manières selon les angles. Pour ces raisons, la sculpture renvoie à la définition d'opera aperta mentionnée plus haut. L'œil ne saisit l'intégralité d'une sculpture que de façon cumulative, une sculpture n'étant que la somme de ses différentes perspectives possibles. La photographie est un instrument qui permet d'immortaliser les perspectives infinies au travers desquelles la sculpture se montre. Cependant, les photographies de Cy Twombly semblent vouloir investir le sujet de possibilités de lecture autres. Si la sculpture est une œuvre dont la compréhension est subordonnée à une intervention active du spectateur, qui est obligé de bouger pour pouvoir en saisir la complexité, les photographies de Twombly ne visent pas à restituer l'œuvre sculptée dans son intégralité. En effet, quand le spectateur fait le tour de l'œuvre sculptée, elle lui apparaît sous plusieurs aspects; une série de photographies prises autour d'une sculpture pourrait éventuellement constituer un support à la compréhension de l'œuvre, en facilitant le processus cumulatif auquel nous sommes confrontés lorsque nous cherchons à reconstruire l'image intégrale d'une sculpture. C'est d'ailleurs dans cette direction, entre pédagogie et documentation, que les photographes en charge dans les départements des musées agissent. En revanche, quand Cy Twombly 
photographie ses sculptures, il ne le fait pas dans le but de les expliquer. Au contraire, la photographie de Twombly permet à la sculpture de s'enrichir de nouvelles significations, par le passage de la troisième à la deuxième dimension et par le fait qu'il ne photographie que rarement les sculptures dans leur intégralité. La relation dialectique entre sculpture et photographie se construit par l'équilibre de forces opposées: déconstruisant l'espace euclidien où la sculpture a été réalisée, la photographie absorbe l'espace tridimensionnel. Le sculpteur insère un fragment de matière dans l'espace, le photographe extrait un moment spatio-temporel de l'espace en tant que fragment.

19 Pour cela, nous avons des raisons de croire que l'aménagement de la salle Lescot a été considéré par Twombly comme entrant en cohérence avec ses recherches esthétiques, d'autant plus que sa fascination pour la culture méditerranéenne s'était renouvelée grâce à plusieurs séjours en Grèce. La salle est caractérisée par l'équilibre des formes, les sculptures dialoguant avec la surface plane du plafond. Twombly relit l'antique et il s'en approprie des éléments: le style du plafond, le choix des couleurs et la fragmentation des boucliers s'inscrivent dans un projet d'appropriation et de transformation de l'antique. Fasciné à la fois par les formes du passé et désireux de dépasser les limites imposées par les règles de composition classiques, l'œuvre de Twombly n'est point un simple hommage à la gloire des ancêtres. Recherche érudite, tension de forces opposées et interactions entre différents médiums caractérisent son œuvre. Le désir d'appropriation et de relecture du passé s'exprime davantage dans ses séries photographiques, car c'est grâce aux possibilités intrinsèques de l'appareil photographique que Twombly pousse sa réflexion esthétique plus loin. Dans la série Sperlonga, exécutée en 2007 dans le Musée archéologique de Sperlonga, à proximité de Gaeta, Twombly tourne son appareil vers la copie romaine d'un groupe sculpté grec représentant l'épopée d'Ulysse. Dans un des clichés les plus représentatifs, Polyphème est photographié en contre-plongée: la lumière tamisée réduit le contraste et l'impression en dry-print donne à la surface du papier un aspect poussiéreux et granuleux. Polyphème n'est pas reconnaissable. Il ne s'agit d'ailleurs que d'un détail de sa figure ; la forte contre-plongée nous donne l'impression d'une figure debout, alors qu'en réalité Polyphème est sculpté allongé sur un rocher en marbre, qui dans la photographie se fond avec le genou au premier plan. Le bâton pointu adressé à l'œil non visible - du cyclope est le seul élément qui nous permet, avec beaucoup d'imagination, de reconnaître Polyphème dans le sujet de ce cliché. Le choix de l'artiste d'exécuter une série de Polaroïds de ce site en 2007 n'est pas une coïncidence. Le site de Sperlonga constitue un exemple de mise en valeur des fouilles archéologiques et de la relation avec leur territoire. Encore une fois, Cy Twombly fait preuve d'une certaine sensibilité à l'archéologie et à l'histoire ancienne, en mettant en valeur ces objets fouillés mais aussi d'autres aspects qui ne ressortent que par l'intermédiaire de la photographie. Il revisite le passé et nous en propose une vision différente. De même, le plafond du Louvre constitue le moment le plus haut de cette incessante recherche d'équilibre entre Antiquité et modernité, entre compréhension des formes anciennes et dépassement de celles-ci en faveur des défis techniques et esthétiques les plus audacieux.

20 Couronnement d'une activité artistique intense et variée, le plafond du Louvre est le testament esthétique de Cy Twombly. Il lègue à l'Europe une œuvre monumentale dans le berceau de l'art occidental et plusieurs centaines de traces plus discrètes, parfois 
imprimées, parfois encore à l'état de Polaroïd, dispersées dans les espaces silencieux de l'atelier de Gaeta, où le temps s'est arrêté à la dernière photographie. La valorisation du fonds photographique de Cy Twombly a permis une analyse plus exhaustive et profonde de son œuvre picturale et sculptée ; la création photographique renouvelle le regard sur ce grand artiste, pour qui elle a constitué un moyen d'expression à part entière. Cela a été possible dans un contexte nouveau d'intérêt pour la photographie des peintres. En effet, les plus grands artistes du vingtième siècle ont souvent recouru à l'appareil photographique; longtemps considérée comme une activité parallèle, la photographie retrouve aujourd'hui une place centrale dans la réflexion sur la création artistique. Twombly fait partie des grands artistes du vingtième siècle pour lesquels la photographie représente à la fois un moyen expressif et un instrument de recherche.

\section{NOTES}

1. Michael Krüger, Cy Twombly, Vol. IV, Unpublished photographs, 1951-2011, Munich, Schirmer/ Mosel, 2012, p. 173.

2. Roland Barthes, "Sagesse de l'art ", dans cat d'exp, David Whitney (dir.), Cy Twombly, painting and drawings, 1954-1977, Whitney Museum of American Art, 10 avril-10 juin 1979, Boston, Michael and Winifred Bixler, 1979, p. 9.

3. Idem, Ibidem, p. 16.

4. William Katz, Cy Twombly Photographs, Matthew Marks Gallery, 15 octobre-4 décembre 1993, New York, Matthew Marks Gallery, 1993.

5. Rosalind Krauss, L'inconscient optique, Paris, Éditions Au même titre, 2002.

6. Hubertus von Amelunxen, "Cette rose ne l'interromps / Qu'à verser un silence pire, photographies de Cy Twombly", dans CyTwombly Photographs Vol. III, 1951-2010, Munich, Schirmer/Mosel, 2011, p. 170.

7. Walter Benjamin, «Petite histoire de la photographie ", Études photographiques, $1^{\mathrm{er}}$ novembre 1996, [En ligne], mis en ligne le 18 novembre 2002, http://etudesphotographiques.revues.org/99 [consulté le 5 juillet 2016].

8. Umberto Eco, Opera aperta, Forma e indeterminazione nelle poetiche contemporanee, Milan, I grandi tascabili Bompiani, 2013.

9. Rosalind Krauss, L'inconscient optique, Paris, Éditions Au même titre, 2002

10. Walter Benjamin, «Petite histoire de la photographie ", Études photographiques, $1^{\text {er }}$ novembre 1996, [En ligne], mis en ligne le 18 novembre 2002, http://etudesphotographiques.revues.org/99 [consulté le 5 juillet 2016]. 


\section{RÉSUMÉS}

L'œuvre photographique de Cy Twombly inclut un vaste nombre de Polaroïds, imprimés avec la technique du dry-print et du tirage Fresson. Bien plus qu'un simple photographe, Cy Twombly est un théoricien de l'art qui questionne les interactions entre peinture et photographie. À travers sa manipulation de la matière, il interroge les limites de l'objet photographique et bouleverse l'univers de la photographie instantanée, qu'il transforme en un objet unique et hybride. Le plafond peint par l'artiste dans la salle des antiquités grecques et romaines du musée du Louvre témoigne de son intérêt pour le dialogue entre photographie, peinture et culture ancienne, et entre en cohérence avec ses recherches esthétiques, notamment en ce qui concerne sa fascination pour la culture méditerranéenne. Loin d'être une simple décoration, le plafond du Louvre pourrait être interprété comme une grande photographie, un morceau fugitif de réalité capturé et circonscrit dans l'espace d'un cadre limité.

Cy Twombly's photographic oeuvre includes a huge number of Polaroids, printed using the dryprint technique and Fresson process. More than just a photographer, Twombly is an art theoretician who examines the interactions between painting and photography. Through his manipulation of the material, he questions the limits of the photographic object and disrupts the world of the instant photograph, which he transforms into a unique, hybrid object. The ceiling painted by the artist in the room of Greek and Roman antiquities in the Louvre bears witness to his interest in the dialogue between photography, painting and American culture, and was consistent with his aesthetic experiments, particularly regarding his fascination for Mediterranean culture. Far from being simply decorative, the Louvre ceiling could be interpreted as a large photograph, a fugitive piece of reality captured and circumscribed in the space of a limited frame.

\section{INDEX}

Keywords : Cy Twombly, Polaroid, dry print, photography, printing, Louvre, contemporary art, Italy, Mediterranean

Mots-clés : Cy Twombly, Polaroïd, dry-print, photographie, impression, Louvre, art contemporain, Italie, Méditerranée

\section{AUTEUR}

\section{CHIARA AGRADI}

Après avoir obtenu un diplôme de licence en Études historiques et artistiques à l'université de Rome La Sapienza, Chiara Agradi a intégré l'École du Louvre, où elle s'est initiée à l'étude de la photographie. Après avoir mené un travail de recherche sur le photojournaliste André Papillon dans le cadre d'une collaboration avec le Cabinet de la photographie du Centre Pompidou, elle a continué à étudier les relations entre ce médium et la peinture, notamment dans le cadre du mémoire de Master 2, consacré à la photographie de $\mathrm{Cy}$ Twombly. Suite à cela, elle a obtenu un master professionnel en Management de l'art et des industries culturelles à HEC, Paris. Elle se destine désormais au monde de la recherche et des institutions culturelles dans le domaine de la photographie.

*** 
After receiving a bachelor's degree in history and art history from the Sapienza-Università di Roma, Chiara Agradi enrolled at the École du Louvre, where she began studying photography. After researching the photojournalist André Papillon as part of a collaboration with the Cabinet de la Photographie of the Centre Pompidou, she continued to study the relationship between photography and painting, notably as part of a Master 2 thesis on Cy Twombly's photography. She then obtained a Master Professionnel in arts management and the cultural industries at HEC, Paris. She now wants to enter the world of research and cultural institutions in the field of photography. 